\title{
ON CLASSICAL AND QUANTUM OBJECTIVITY
}

\author{
GABRIEL CATREN \\ CREA, UMR 7656, ÉCOLE POLYTECHNIQUE/CNRS, 1 RUE DESCARTES, \\ 75005, PARIS, FRANCE
}

\begin{abstract}
We propose a conceptual framework for understanding the relationship between observables and operators in mechanics. To do so, we introduce a postulate that establishes a correspondence between the objective properties permitting to identify physical states and the symmetry transformations that modify their gauge dependant properties. We show that the uncertainty principle results from a faithful - or equivariant - realization of this correspondence. It is a consequence of the proposed postulate that the quantum notion of objective physical states is not incomplete, but rather that the classical notion is overdetermined.
\end{abstract}

\section{INTRODUCTION}

It is commonly stated that quantum mechanics differs from classical mechanics in its use of operators acting on physical states. According to this description, the transition from classical to quantum mechanics can be understood as a substitution of a commutative algebra of functions - relative to pointwise multiplication - by a non-commutative algebra of operators. Nevertheless, the use of operators acting on physical states is also an essential feature of classical mechanics. In fact, classical observables play two fundamental roles in mechanics, namely they are functions that can be evaluated on states - and used for identifying them - and they define Hamiltonian vector fields that act on states by means of infinitesimal canonical transformations (see Refs.[1], [2], [5], [9], [10], [13]). For example, the temporal evolution of classical systems is given by the integration of the infinitesimal classical action generated by the Hamiltonian vector field $v_{H}$ associated to the Hamiltonian function $H(q, p)$. Nevertheless, the Poisson algebra of classical observables and the Lie algebra of classical operators (under the Lie bracket of vector fields) are not isomorphic (given that the corresponding map is not injective). This means that classical mechanics fails 
to establish a faithful correspondence between the two fundamental roles played by classical observables. According to Dirac's quantization conditions [4], quantum mechanics can be partially obtained by forcing an isomorphism between the algebraic structures associated to these two roles, namely between a subalgebra of the Poisson algebra of classical observables and the commutator algebra of operators. In the framework of the geometric quantization program, this task can be achieved by extending classical operators $v_{f}$ - Hamiltonian vector fields associated to observables $f$ - to quantum operators $\hat{v}_{f}$, such that the commutator algebra of the latter is isomorphic to the Poisson algebra of classical observables. This quantum correction of classical operators can be performed by defining a complex line bundle $L \rightarrow M$ over the phase space $M$, endowed with a hermitian connection such that its curvature is given by the symplectic form on $M$. The polarized sections of this complex line bundle define the corresponding quantum states (see Refs.[3], [8], [12], [13], [14]).

The fact that quantum mechanics can be obtained by establishing a faithful correspondence between observables and operators suggests that a deeper analysis of the conceptual meaning of this correspondence could shed some new light on the rational necessity of quantum mechanics. In what follows, we will propose a conceptual framework for understanding the relation between observables and operators. We will argue that their correspondence can be understood in terms of the relation between the objective properties that define physical states and the symmetry transformations that interchange their gauge dependant properties. To do so, we will show that, even tough it remains valid, the standard characterization of objective properties as the invariants under symmetry transformations does not suffice for properly understanding the correspondence between observables and operators. Therefore, we will propose a postulate that specifies the lacking fundamental aspect of their relation. Roughly speaking, we will argue that the objective properties of a physical state play a twofold role, namely 1) they permit to identify the physical state, and 2) they induce the transformations between the different "aspects", "profiles" or non-objective properties of the state. From a conceptual point of view, we will then show that the proposed postulate implies the necessity of an uncertainty principle. According to this postulate, the quantum description of a physical state is not underdetermined, but rather 
the classical description is overdetermined. This implies that quantum mechanics should not be considered an obstruction to the validity of a well-defined classical notion of objectivity, but rather as a consistent theory of physical objectivity that overcomes the classical impasses. The quantum uncertainty principle can be considered the signature of this achievement.

In this work we analyze the rational necessity of quantum mechanics by proposing a new interpretation of one of its fundamental features, namely the uncertainty principle. In Sect. II, we propose a postulate - not satisfied by classical mechanics - in order to understand the fundamental correspondence between observables and operators. In Sect. III, we show that this postulate can be further clarified and justified by giving a precise definition to the notion of physical states' possible properties. To do so, we consider the so-called momentum map as well as its equivariance properties. We show that the failure of classical mechanics to establish a faithful correspondence between observables and operators is manifested through the non-equivariance of the relevant momentum map. We then consider how quantum mechanics can be obtained by forcing such an equivariance. In Sect. IV, we consider a dual version of this last result by using the geometric quantization formalism. In Sect. V, we summarize and discuss the main results.

\section{OBJeCtive PhysicAl StATES}

In general, the relevant variables allowing us to specify a physical state depend on the arbitrary election of a coordinate system. The objective properties of the state are then identified with the invariants under the symmetry transformations that modify the coordinate systems. In this section, we argue that this standard characterization of objective properties does not suffice for understanding the fundamental role played by symmetry transformations in classical and quantum mechanics.

In classical mechanics, the transformations of the canonical variables used for specifying a physical state are given by canonical transformations. In particular, we are interested in considering infinitesimal canonical transformations induced by classical observables. In fact, the symplectic structure $\omega$ of the phase space $M$ permits to pass from an observable $f \in \mathcal{C}^{\infty}(M)$ to the symplectic diffeomorphism $\phi_{\lambda}^{f}: M \rightarrow M$ 
generated by the Hamiltonian vector field $v_{f}$ induced by $f$. The relation between the observable and the induced Hamiltonian vector field $v_{f}$ is given by the expression $i_{v_{f}} \omega=d f$, where $i_{v_{f}} \omega$ denotes the contraction of the 2 -form $\omega$ with the vector $v_{f}$. ${ }^{1}$ Therefore, the classical observables $f \in \mathcal{C}^{\infty}(M)$ play a twofold role. Not only do they define local coordinates on the phase space $M$ - which can be used for identifying classical states -, but they also induce infinitesimal canonical transformations. In principle, these transformations might be equivalently interpreted either as passive transformations of the coordinate system or as active transformations of physical states [5]. The formal equivalence between passive and active transformations will be called Leibniz equivalence. In general relativity, the invariance under the group of general diffeomorphisms of space-time implies that - if Leibniz equivalence is accepted localization on space-time is pure gauge. In principle, we might think that the invariance of classical mechanics under the group of simplectic diffeomorphisms can be interpreted in an analogous way. However, such is not the case. If Leibniz equivalence were valid, we would be forced to accept that localization on $M$ is pure gauge. But, in that case the very notion of a space that parameterizes physically distinct states would be lost. Consequently, infinitesimal canonical transformations induced by classical observables are interpreted as active transformations between physically distinct states. For example, the canonical transformations induced by the Hamiltonian function $H(q, p) \in \mathcal{C}^{\infty}(M)$ generates the temporal evolution of physical states, that is to say their objective physical change in time. In other words, "[...] the motion of a mechanical system corresponds to the continuous evolution or unfolding of a canonical transformation." [5]. More generally, through its induced Hamiltonian vector field $v_{p}=\partial_{q}$, a momentum $p$ generates canonical transformations of the conjugated coordinate $q$. Since $H$ generates temporal evolutions of any observable and $p$ generates canonical transformations of $q$, the temporal evolution of $q$ is given by the dependance of $H$ on $p$ (through the Hamilton's equation $\left.\dot{q}=\frac{\partial H}{\partial p}\right){ }^{2}$ Since the temporal evolution of $q$ is considered an effective physical change, general

\footnotetext{
${ }^{1}$ In $M=\mathbf{R}^{2}$, the Hamiltonian vector field associated to an observable $f \in$ $\mathcal{C}^{\infty}(M)$ is given by the expression $v_{f}=\frac{\partial f}{\partial p} \frac{\partial}{\partial q}-\frac{\partial f}{\partial q} \frac{\partial}{\partial p}$.

${ }^{2}$ This remark explains the fundamental conceptual difference between $\dot{q}$ (the infinitesimal temporal evolution of $q$ ) and $p$ (the observable that generates infinitesimal canonical transformations of $q$ ).
} 
transformations generated by $p$ should also be interpreted as active changes of the state. ${ }^{3}$

Classical mechanics is thus characterized by its denial of Leibniz equivalence: states related by canonical transformations induced by classical observables are considered to be physically distinct states. In what follows we argue that, in order to improve the comprehension of the foundations of mechanics, it is necessary to partially maintain the validity of Leibniz equivalence. Moreover, quantum mechanics is the formalism required to satisfy this prescription. According to this interpretative scheme, we will consider states connected through certain canonical transformations induced by physical observables to be gauge equivalent. This implies that the particular value of the coordinate affected by the canonical transformation is not an objective property of the state. In other words, localization in the orbit of the corresponding symplectic diffeomorphism is pure gauge. We will call objective reduction the identification of states by means of certain infinitesimal canonical transformations.

As we have seen, if we considered all possible infinitesimal canonical transformations as symmetry transformations, all states in $M$ would be gauge equivalent. It is then necessary to specify the particular subset of infinitesimal canonical transformations that should be considered for performing the objective reduction of each state. To do so, we propose the following fundamental postulate:

† The objective properties of a physical state define the only generators of symmetry transformations accepted for its objective reduction.

A set of objective properties that, according to this postulate, defines both the identity of an objective physical state $s$ and the correct symmetry transformations needed for its objective reduction, will be called eidos $\varepsilon_{s}$ of the physical state $s$. In this way, the eidos that identifies a particular physical state also specifies which properties should not be considered objective properties but "profiles" that depend on the coordinate system.

In classical mechanics the set of objective properties of a physical state is given by the $2 n$ local coordinates $\left(q^{i}, p_{i}\right)_{i=1, \ldots, n}$ of the phase

\footnotetext{
${ }^{3}$ For instance, the uniform rotation generated by a conserved angular momentum $\mathbf{L}$ is an effective physical transformation of the system.
} 
space $M$ (in what follows we will restrict the analyses to the case $M=\mathbf{R}^{2 n}$; for the consideration of more general cases see Ref.[6]). According to postulate $\ddagger$, objective properties are in correspondence with the generators of the symmetry transformations required for the objective reduction of the state. In classical mechanics, this correspondence is provided by the map $f \mapsto v_{f}$ between classical observables $f \in \mathcal{C}^{\infty}(M)$ and Hamiltonian vector fields $v_{f} \in \mathcal{H}_{M}$. In particular, the momentum $p$ defines the generator $v_{p}=\partial_{q}$ of infinitesimal translations of the conjugated coordinate $q\left(\mathcal{L}_{i} e_{v_{p}} q=v_{p}(q)=\{q, p\}=1\right)$ and viceversa. Since in classical mechanics both $q$ and $p$ are used for identifying the state, postulate $\ddagger$ demands that both $v_{q}$ and $v_{p}$ should be used for the objective reduction of the corresponding state. But this implies that both $q$ and $p$ are gauged out, which is contradictory with the assumption that they define objective properties of the state (since by definition objective properties have to be invariant under symmetry transformations). In other words, the action defined by both $v_{q}$ and $v_{p}$ reduces the phase space $M$ to a single point $\{*\}$ or, equivalently, the group $G$ of translations in both position and momentum acts transitively on $M$ (i.e. $M$ is a single $G$-orbit). Clearly, this is a reductio ad absurdum. We began by supposing that a physical state is identified by means of the $2 n$ coordinates of the phase space $M$, that is to say that each point in $M$ defines a different physical state. We nevertheless found, guided by postulate $\ddagger$, that there is only one possible physical state $\{*\}$. We can thus conclude that objective physical states defined by an eidos that satisfies postulate $\ddagger$ cannot be identified with classical states in $M$.

This classical failure to implement postulate $\ddagger$ is a direct consequence of the fact that classical states are specified by means of too many variables. We will refer to this fact as the classical overdetermination of the eidos. According to postulate $\ddagger$, an objective property $f \in \varepsilon_{s}$ of the physical state $s$ has to be invariant under the infinitesimal canonical transformations generated by the whole set of objective properties in $\varepsilon_{s}$. This implies that $v_{g} f=\{f, g\}=0$ for all $f, g \in \varepsilon_{s}$. Therefore, the set of observables in the eidos $\varepsilon_{s}$ have to define a commutative Poisson algebra. Hence, an eidos that completely specifies an objective physical state is given by the maximal number of mutually commuting observables (i.e. by a complete set of commuting observables). In order to satisfy postulate $\ddagger$ it is then necessary to reduce the eidos to 
half the number of components. Therefore, an acceptance of the proposed postulate implies that the quantum notion of objective physical states is not incomplete (when compared to a hypothetical complete classical description), but rather that the classical notion is overdetermined. As a consequence of this overdetermination of classical states, the relationship established between objective properties and symmetry transformations by postulate $\ddagger$ cannot be elucidated within the framework of classical mechanics. In fact, the assumption of postulate $\ddagger$ implies what we could call the a priori necessity of an uncertainty principle. If an objective physical state is identified by means of an eidos composed of $n$ components, there should be $n$ other possible properties that are gauged out. This means that the values of the latter have no objective physical meaning. For example, the momentum $p$ of a system with a well-defined position $q$ is completely gauged out by the symmetry transformation induced by the objective property $q$. Hence, the momentum $p$ is necessarily undetermined. Therefore, the possible properties of an objective physical state $s$ can be separated into two sets: the $n$ objective properties $\left\{x_{i}\right\}_{i=1, \ldots n}$ belonging to the eidos $\varepsilon_{s}$, and the $n$ non-objective properties $\left\{x_{j}\right\}_{j=n+1, \ldots, 2 n}$ that are gauged out by the symmetry transformations defined by the former. The fact that there is an even number of possible properties is a direct consequence of this intertwining between objective and non-objective properties. Therefore, the set of possible properties has to be given by a twofold set of $2 n$ symplectically intertwined - or canonically conjugated - properties.

Since general canonical transformations mix canonical variables, it should be possible to have properties which are neither objective properties nor profiles, but a mixture of both. In fact, the flexibility of quantum mechanics' formalism enables the consideration of intermediate physical states where neither $q$ nor $p$ are sharp objective properties. If for example $q$ is an unsharp objective property of a physical state, the conjugated momentum $p$ is not completly gauged out. Hence $p$ is in turn an unsharp objective property that partially gauges the coordinate $q$. This means that, for a given physical state, a certain property can be partially considered an unsharp objective property (that partially gauges the conjugated variable) and partially a gauged variable. The resulting subtle equilibrium between unsharp objective properties and 
unsharp non-objective profiles are formally governed by the uncertainty principle.

\section{From UNIVERSAL SYMMETRY TRANSFORMATIONS TO OBJECTIVE PROPERTIES}

Postulate $\ddagger$ establishes a conceptual correspondence between the objective properties of a physical state and the induced symmetry transformations that connect its gauge dependant "profiles". In this section we will justify the necessity of such a correspondence by giving a precise meaning to the notion of physical states' properties. To do so, we will consider the transition from a group of symmetry transformations acting on states to the definition of certain quantities - the momenta - that define the possible properties of the states. This relation is provided by the so-called momentum map (see Refs.[9], [10], [11]). In other words, instead of going from objective properties to (realized) symmetry transformations - by means of the map $f \mapsto v_{f}-$, we will go from (universal) symmetry transformations to objective properties. Under certain conditions, the momentum map permits to define the objective properties of a physical state as quantities that specify how the physical state realizes in a particular way certain universal operations. As we will show, these conditions are not satisfied in classical mechanics.

The action $\Phi: G \times M \rightarrow M$ of a Lie group $G$ on a manifold $M$ defines a map $\iota$ between Lie algebra elements $\xi \in \mathrm{g}$ and fundamental vector fields $v_{\xi}$ on $M$. This map is defined by means of the expression $v_{\xi}(x)=$ $\left.\frac{d}{d \lambda}(\exp (-\lambda \xi) \cdot x)\right|_{\lambda=0}$, for $x \in M$. At each $x \in M$, the fundamental vector field $v_{\xi}$ generates the infinitesimal transformation of the classical state $x$ defined by the universal Lie algebra element $\xi \in \mathrm{g}$. We will say that $v_{\xi}(x) \in T_{x} M$ is the realization by the particular state $x$ of the universal infinitesimal generator $\xi$. The action $\Phi$ is said to be symplectic if $G$ acts on $M$ by means of symplectic diffeomorphisms, i.e. if $\Phi_{g}^{*} \omega=\omega$ for all $g \in G$ (where $\Phi_{g}:=\Phi(g, \cdot): M \rightarrow M$ ). The relevant question is whether it is possible to obtain the fundamental vector field $v_{\xi}$ as a Hamiltonian vector field corresponding to a function $h_{\xi} \in \mathcal{C}^{\infty}(M)$. If this is the case, the fundamental vector field $v_{\xi}$ has to satisfy the equation $i_{v_{\xi}} \omega=d h_{\xi}$. A symplectic $G$-action is said to be 
Hamiltonian if there exists a map

$$
\begin{aligned}
\tilde{\mu}: \mathrm{g} & \rightarrow \mathcal{C}^{\infty}(M) \\
\xi & \mapsto h_{\xi}
\end{aligned}
$$

(called co-momentum) such that the following diagram commutes

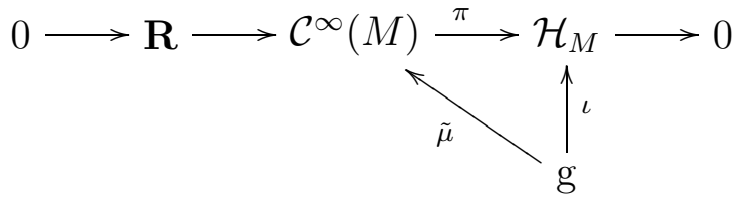

where the short sequence is exact. ${ }^{4}$ The co-momentum map $\tilde{\mu}$ is defined up to a constant, i.e. the function $\tilde{\mu}^{\prime}(\xi)=h_{\xi}+k$ (with $k \in \mathbf{R}$ ) has also $v_{\xi}$ as its induced Hamiltonian vector field. The corresponding momentum map is the map

$$
\mu: M \rightarrow \mathrm{g}^{*},
$$

defined by

$$
\langle\mu(x), \xi\rangle=\tilde{\mu}(\xi)(x)=h_{\xi}(x),
$$

with $\xi \in \mathrm{g}$ and $\langle\cdot, \cdot\rangle: \mathrm{g}^{*} \times \mathrm{g} \rightarrow \mathbf{R}$ the natural duality pairing. ${ }^{5}$ Thanks to these maps, the realization of universal operations in $\mathrm{g}$ as fundamental vector fields on $M$ can be factorized through functions in $\mathcal{C}^{\infty}(M)$. As we will see, if certain conditions are satisfied - namely if the momentum map is equivariant and if the map $\mathcal{C}^{\infty}(M) \rightarrow \mathcal{H}_{M}$ is injective -, then physical observables can be defined as quantities that specify how different physical states realize differently universal operations in g. The important fact is that these conditions are no satisfied in classical mechanics.

For a fixed $x \in M, \mu(x)$ is an element in $\mathrm{g}^{*}$ that takes on each infinitesimal generator $\xi \in \mathrm{g}$ a certain value $\langle\mu(x), \xi\rangle \in \mathbf{R}$ that depends, by definition, on the state $x$. The quantity $\langle\mu(x), \xi\rangle$ can then be used

\footnotetext{
${ }^{4}$ The exactness of this sequence simply means that the image of the inclusion $\mathbf{R} \rightarrow \mathcal{C}^{\infty}(M)$ - the constant functions $f=k \in \mathbf{R}$ in $\mathcal{C}^{\infty}(M)$ - coincides with the kernel of the projection $\pi$. In other terms, $v_{f=k}=0$.

${ }^{5}$ The ambiguity in the co-momentum map $\tilde{\mu}$ induces an ambiguity in the corresponding momentum map. The modified momentum map $\mu^{\prime}$ has to satisfy $\left\langle\mu^{\prime}(x), \xi\right\rangle=\tilde{\mu}^{\prime}(\xi)(x)=\tilde{\mu}(\xi)(x)+k$. If one defines $\mu^{\prime}(x)=\mu(x)+a$ with $a \in \mathrm{g}^{*}$ such that $a(\xi)=k$, this relation is satisfied. This means that the momentum map is defined up to a constant in $\mathrm{g}^{*}$.
} 
for identifying - at least partially - the state $x$. By considering how this quantity depends on the states, this information can be used to distinguish between states. To do so, one can fix a universal generator $\xi \in \mathrm{g}$ and compare the values of $\langle\mu(x), \xi\rangle$ for different states $x$. In fact, each element $\xi \in \mathrm{g}$ defines a function $h_{\xi}: M \rightarrow \mathbf{R}$ (called a momentum) given by $h_{\xi}(x)=\langle\mu(x), \xi\rangle$. By construction, the Hamiltonian vector field associated to the momentum $h_{\xi}$ is the fundamental vector field $v_{\xi}$ that realizes the universal transformation $\xi$. For example, the angular momentum $\mathbf{L}(\mathbf{q}, \mathbf{p})$ of a particle in Euclidean space is given by the momentum map $\mu(\mathbf{q}, \mathbf{p}):=\mathbf{L}(\mathbf{q}, \mathbf{p})=\mathbf{q} \times \mathbf{p}$. If one selects a particular Lie algebra element $\xi \in \mathrm{g}=\mathbf{R}^{3}$, the function $L_{\xi}: M \rightarrow \mathbf{R}$ given by $=L_{\xi}(\mathbf{q}, \mathbf{p})=\langle\mu(\mathbf{q}, \mathbf{p}), \xi\rangle=\xi \cdot(\mathbf{q} \times \mathbf{p})$ defines a possible property of classical states in $M$. The Hamiltonian vector field $v_{L_{\xi}}$ associated to the observable $L_{\xi}$ generates canonical transformations that can be identified with rotations around the axis defined by $\xi$.

If the co-momentum map $\tilde{\mu}$ is a homomorphism of Lie algebras i.e. if $\tilde{\mu}([\xi, \eta])=\{\tilde{\mu}(\xi), \tilde{\mu}(\eta)\}-$, the symplectic $G$-action is said to be strongly Hamiltonian. It can be shown that this is the case if and only if the momentum map is infinitesimally equivariant, i.e. if $T_{x} \mu\left(v_{\xi}(x)\right)=$ $-a d_{\xi}^{*} \mu(x)$ for all $\xi \in \mathrm{g}$, where $T_{x} \mu: T_{x} M \rightarrow T \mathrm{~g}^{*} \simeq \mathrm{g}^{*}$ and $a d^{*}$ denotes the coadjoint action of $\mathrm{g}$ on $\mathrm{g}^{*}$ (see Refs.[10], [11]). ${ }^{6}$ The action of a group $G$ on the manifold $M$ is equivariant if $A d_{g^{-1}}^{*} \circ \mu=\mu \circ \Phi_{g}$ (where $A d^{*}$ denotes the coadjoint action of $G$ on $\mathrm{g}^{*}$ ), i.e. if the following diagram commutes

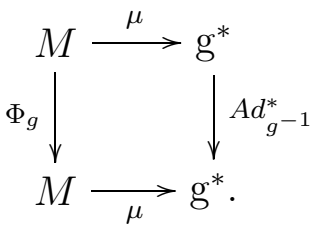

It can be shown that equivariance implies infinitesimal equivariance and that the converse is true only if $G$ is connected [10]. For compact

\footnotetext{
${ }^{6}$ The adjoint representation of a Lie group $G$ on its Lie algebra $\mathrm{g}$ is defined by $A d_{g}:=T_{e} I_{g}: \mathrm{g} \rightarrow \mathrm{g}$, where $I_{g}: G \rightarrow G$ is given by $I_{g}(h)=g h g^{-1}$. The infinitesimal adjoint action of $\mathrm{g}$ on $\mathrm{g}$ is defined by the map $a d_{\xi}: \mathrm{g} \rightarrow \mathrm{g}$ given by $a d_{\xi} \eta=[\xi, \eta]$ with $\xi, \eta \in \mathrm{g}$. The coadjoint action of $G$ on $\mathrm{g}^{*}$ is defined by using the dual map $A d_{g}^{*}: \mathrm{g}^{*} \rightarrow \mathrm{g}^{*}$ given by $\left\langle A d_{g}^{*} \mu, \xi\right\rangle=\left\langle\mu, A d_{g}(\xi)\right\rangle$, with $\mu \in \mathrm{g}^{*}$ and $\xi \in \mathrm{g}$. The coadjoint action of $G$ on $\mathrm{g}^{*}$ is then defined by the map $\Phi^{*}: G \times \mathrm{g}^{*} \rightarrow \mathrm{g}^{*}$ given by $(g, \mu) \mapsto A d_{g^{-1}}^{*} \mu$. The infinitesimal coadjoint action of $\mathrm{g}$ on $\mathrm{g}^{*}$ is defined by the map $a d_{\xi}^{*}: \mathrm{g}^{*} \rightarrow \mathrm{g}^{*}$ given by the expression $a d_{\xi}^{*}(\mu)=\langle\mu,[\xi, \cdot]\rangle[10]$.
} 
Lie groups, one can always use the fact that momentum maps are defined up to a constant in $\mathrm{g}^{*}$ in order to choose them as equivariant [11].

We will now consider the significance of this equivariance property. The important result is that, for equivariant momentum maps, the dual $\mathrm{g}^{*}$ of the Lie algebra $\mathrm{g}$ defines a final object in the category of Poisson $G$-manifolds, the momentum map $\mu$ being the unique morphism from an object in the category - i.e. a phase space $M$ - to the universal object $\mathrm{g}^{*}[7]$. A Poisson $G$-manifold is a pair $(M, \tilde{\mu})$, where $M$ is a Poisson manifold with a $G$-action and $\tilde{\mu}_{M}$ a strong co-momentum map, i.e. a homomorphism of Lie algebras $\tilde{\mu}_{M}: \mathrm{g} \rightarrow \mathcal{C}^{\infty}(M)$. It is then possible to define the category $\mathcal{G}$ of all Poisson $G$-manifolds, where a morphism $\alpha:\left(M, \tilde{\mu}_{M}\right) \rightarrow\left(N, \tilde{\mu}_{N}\right)$ is a smooth map from $M$ to $N$ which preserves the Poisson bracket structure (i.e. which satisfies $\left.\alpha^{*}\{f, g\}=\left\{\alpha^{*} f, \alpha^{*} g\right\}\right)$ and such that the following diagram commutes

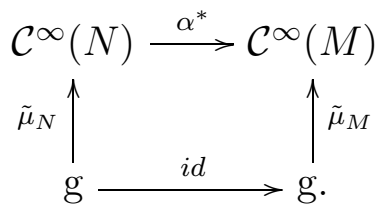

It can be shown that the dual vector space $\mathrm{g}^{*}$ of the Lie algebra $\mathrm{g}$ defines a final object in this category. The action of $G$ on $\mathrm{g}^{*}$ is the coadjoint action. Under this action, $\mathrm{g}^{*}$ breaks up into so-called coadjoint orbits. ${ }^{7}$ The vector space $\mathrm{g}^{*}$ has a canonical Poisson bracket structure. This means that $\mathrm{g}^{*}$ is a Poisson manifold, with the coadjoint orbits as its symplectic leaves. The Poisson bracket on $\mathcal{C}^{\infty}\left(\mathrm{g}^{*}\right)$ can be defined as follows. If $f, g \in \mathcal{C}^{\infty}\left(\mathrm{g}^{*}\right)$, then $d f(x), d g(x) \in T_{x}^{*} \mathrm{~g}^{*} \simeq \mathrm{g}$ (for $x \in \mathrm{g}^{*}$ ). The Poisson bracket can then be defined as $\{f, g\}(x)=$ $\langle x,[d f(x), d g(x)]\rangle$. In the Poisson $G$-manifold $\mathrm{g}^{*}$, it is possible to define

\footnotetext{
${ }^{7}$ The coadjoint orbit through $\mu \in \mathrm{g}^{*}$ is the subset of $\mathrm{g}^{*}$ defined by $\mathcal{O}_{\mu} \equiv G \cdot \mu:=$ $\left\{A d_{g^{-1}}^{*}(\mu), \forall g \in G\right\}$. The fundamental property of coadjoint orbits is that they are symplectic manifolds. In order to define the corresponding symplectic structures let's consider the tangent vectors to coadjoint orbits. If $\mu(t)=A d_{g(t)^{-1}}^{*} \mu$ is a curve in $\mathcal{O}_{\mu}$ with $\mu(0)=\mu$ and $g(t)=\exp (t \xi)$ for $\xi \in \mathrm{g}$, it can be shown that $\mu^{\prime}(0)=-a d_{\xi}^{*} \mu$. Thus $T_{\mu} \mathcal{O}_{\mu}=\left\{a d_{\xi}^{*} \mu, \xi \in \mathrm{g}\right\}$. The symplectic structure on $\mathcal{O}_{\mu}$ can be defined as $\omega_{\mathcal{O}_{\mu}}(\mu)\left(-a d_{\xi}^{*} \mu,-a d_{\eta}^{*} \mu\right)=\langle\mu,[\xi, \eta]\rangle$. It can be shown that the map $A d_{g^{-1}}^{*}: \mathcal{O}_{\mu} \rightarrow \mathcal{O}_{\mu}$ preserves $\omega_{\mathcal{O}_{\mu}}$ (see Ref.[10] for more details).
} 
a co-momentum map $\tilde{\mu}_{\mathrm{g}^{*}}: \mathrm{g} \rightarrow \mathcal{C}^{\infty}\left(\mathrm{g}^{*}\right)$ given by $\tilde{\mu}_{\mathrm{g}^{*}}(\xi)(x)=\langle x, \xi\rangle .^{8}$ Therefore, the pair $\left(\mathrm{g}^{*}, \tilde{\mu}_{\mathrm{g}^{*}}\right)$ is a Poisson $G$-manifold. Moreover, it is a final object in the category $\mathcal{G}$ of Poisson $G$-manifolds, being the strong momentum map $\mu: M \rightarrow \mathrm{g}^{*}$ the unique morphism from an object $\left(M, \tilde{\mu}_{M}\right)$ to $\left(\mathrm{g}^{*}, \tilde{\mu}_{\mathrm{g}^{*}}\right)$. In this way, the momentum map $\mu: M \rightarrow \mathrm{g}^{*}$ establishes an identification between states in $M$ and elements of the universal model g* for the $G$-action. ${ }^{9}$ Since $G$ also acts on $g^{*}$, the characterization of a mechanical system as a realization of the universal model $\mathrm{g}^{*}$ is valid only if the momentum map $\mu: M \rightarrow \mathrm{g}^{*}$ respects the structure defined by the $G$-action. In other words, the $G$-action has to be equivariant. This means that the realization of the universal model established by the momentum map commutes with the $G$-action on both $M$ and $\mathrm{g}^{*}$. The fact that an equivariant momentum map preserves the relevant structures when passing from the universal model g* to its realization by $M$ is also manifested by the fact that such a map is a Poisson map, i.e. it satisfies $\mu^{*}\{f, g\}=\left\{\mu^{*} f, \mu^{*} g\right\}$, for $f, g \in \mathcal{C}^{\infty}\left(\mathrm{g}^{*}\right)$.

To sum up, we can say that universal operators in g act on concrete states in $M$ by means of realized operators in $\mathcal{H}_{M}$ (fundamental or Hamiltonian vector fields). This realization is mediated by classical observables by means of the co-momentum map $\tilde{\mu}: \mathrm{g} \rightarrow \mathcal{C}^{\infty}(M)$. Since by definition the Lie algebra $g$ acts on its dual $g^{*}$, it is natural to discover that $\mathrm{g}^{*}$ constitues a universal model of the phase space $M$. This means that $\mathrm{g}^{*}$ is a final object in the category of Poisson $G$-manifolds. The identification between the phase space $M$ and the universal model $\mathrm{g}^{*}$ is given by the momentum map $\mu: M \rightarrow \mathrm{g}^{*}$. This correspondence is valid only if the maps respect the relevant algebraic structures. This means that the $G$-action on both $M$ and $\mathrm{g}^{*}$ and the Poisson structures have to be preserved by the corresponding maps.

We will now consider a potential obstruction to the momentum map's infinitesimal equivariance. This obstruction measures the extent to

\footnotetext{
${ }^{8}$ It can be verified that the corresponding momentum map is the identity. Using that $\left\langle\mu_{\mathrm{g}^{*}}(x), \xi\right\rangle=\tilde{\mu}_{\mathrm{g}^{*}}(\xi)(x)=\langle x, \xi\rangle$, it follows that $\mu_{\mathrm{g}^{*}}(x)=x$.

${ }^{9}$ In particular, a coadjoint orbit can be considered the universal model for the orbits of the $G$-action. It can be shown that the image of $\mu$ is a union of coadjoint orbits. In particular, if $G$ acts transitively on $M$, then the momentum map is a covering of a unique coadjoint orbit.
} 
which the co-momentum map $\tilde{\mu}: \mathrm{g} \rightarrow \mathcal{C}^{\infty}(M)$ fails to be a homomorphism of Lie algebras. The obstruction is then given by the map

$$
\Sigma: \mathrm{g} \times \mathrm{g} \rightarrow \mathbf{R}
$$

defined by

$$
\Sigma(\xi, \eta)=\tilde{\mu}([\xi, \eta])-\{\tilde{\mu}(\xi), \tilde{\mu}(\eta)\} .
$$

By using Jacobi's identity in both $\{\cdot, \cdot\}$ and $[\cdot, \cdot]$, it can be shown that $\Sigma$ satisfies

$$
\Sigma(\xi,[\eta, \zeta])+\Sigma(\eta,[\zeta, \xi])+\Sigma(\zeta,[\xi, \eta])=0
$$

This means that $\Sigma$ defines a 2-cocycle in the Lie algebra cohomology $H^{*}(\mathrm{~g}, \mathbf{R})$ of $\mathrm{g}$ with values in $\mathbf{R} .{ }^{10}$ The corresponding cohomology class $[\Sigma] \in H^{2}(\mathrm{~g}, \mathbf{R})$ is the obstruction to the infinitesimal equivariance of the momentum map. The cocycle $\Sigma$ is a coboundary if there exists a 1-cochain $\lambda: \mathrm{g} \rightarrow \mathbf{R}$ such that $\Sigma(\xi, \eta)=\left(\delta_{\mathrm{g}} \lambda\right)(\xi, \eta)=-\lambda([\xi, \eta])$. Two co-momentum maps differing in a constant element $\lambda \in \mathrm{g}^{*}$ have associated cocycles differing in a coboundary: $\Sigma_{\lambda}(\xi, \eta)=\Sigma(\xi, \eta)+$ $\lambda([\xi, \eta])$. This means that they belong to the same cohomology class: $\left[\Sigma_{\lambda}\right]=[\Sigma]$. If the cohomology class is trivial - i.e. if $[\Sigma]=0$ or equivalently $\Sigma(\xi, \eta)=-\lambda([\xi, \eta])-$, then the momentum map can be modified so as to be infinitesimally equivariant. In fact, the new map $\tilde{\mu}^{\prime}=\tilde{\mu}+\lambda$ is an infinitesimally equivariant co-momentum map. ${ }^{11}$ In

\footnotetext{
${ }^{10}$ Let $C^{k}(\mathrm{~g}, \mathbf{R})=\left\{\alpha: \mathrm{g}^{k} \rightarrow \mathbf{R}\right\}$ be the set of $\mathbf{R}$-valued $k$-cochains (where the maps $\alpha$ are skew $k$-linear maps). Let's define the differential $\delta_{\mathrm{g}}: C^{k}(\mathrm{~g}, \mathbf{R}) \rightarrow$ $C^{k+1}(\mathrm{~g}, \mathbf{R})$ as

$$
\left(\delta_{\mathrm{g}} \alpha\right)\left(\xi_{0}, \ldots, \xi_{k}\right)=\sum_{0 \leq i<j \leq k}(-1)^{i+j} \alpha\left(\left[\xi_{i}, \xi_{j}\right], \xi_{0}, \ldots, \hat{\xi}_{i}, \ldots, \hat{\xi}_{j}, \ldots, \xi_{k}\right),
$$
}

where $\hat{\xi}_{i}$ means that $\xi_{i}$ has been suppressed. It can be shown that $\delta_{\mathrm{g}}^{2}=0$. The corresponding cohomology $H^{*}(\mathrm{~g}, \mathbf{R})=\operatorname{ker}\left(\delta_{\mathrm{g}}^{*}\right) / i m\left(\delta_{\mathrm{g}}^{*-1}\right)$ is the Lie algebra cohomology of $\mathrm{g}$ with values in $\mathbf{R}$. In our case, the element $\Sigma$ is a skew bilinear map that satisfies $\left(\delta_{\mathrm{g}} \Sigma\right)(\eta, \xi, \zeta)=-(\Sigma([\eta, \xi], \zeta)+\Sigma([\zeta, \eta], \xi)+\Sigma([\xi, \zeta], \eta))=0$, i.e. it is a 2-cocycle in $H^{2}(\mathrm{~g}, \mathbf{R})$.

${ }^{11}$ The obstruction associated to the co-momentum map $\tilde{\mu}^{\prime}=\tilde{\mu}+\lambda$ is

$$
\begin{aligned}
\Sigma^{\prime}(\xi, \eta) & =\tilde{\mu}^{\prime}([\xi, \eta])-\left\{\tilde{\mu}^{\prime}(\xi), \tilde{\mu}^{\prime}(\eta)\right\} \\
& =\tilde{\mu}([\xi, \eta])+\lambda([\xi, \eta])-\{\tilde{\mu}(\xi)+\lambda(\xi), \tilde{\mu}(\eta)+\lambda(\eta)\} \\
& =\tilde{\mu}([\xi, \eta])+\lambda([\xi, \eta])-\{\tilde{\mu}(\xi), \tilde{\mu}(\eta)\} \\
& =\Sigma(\xi, \eta)+\lambda([\xi, \eta]) \\
& =0 .
\end{aligned}
$$


particular, if $\mathrm{g}$ is semisimple, then the Second Whitehead Lemma states that $H^{2}(\mathrm{~g}, \mathbf{R})=0$.

When the cohomology class of $\Sigma$ is not trivial, the momentum map can still be adjusted to be infinitesimally equivariant by enlarging g to the central extension $\mathrm{g}^{\prime}=\mathrm{g}+\mathbf{R}$ defined by $\Sigma$. The bracket in $\mathrm{g}^{\prime}$ is defined by

$$
[(\xi, a),(\eta, b)]=([\xi, \eta], \Sigma(\xi, \eta)) .
$$

The Lie algebra $g^{\prime}$ acts on $M$ by $\rho(\xi, a)(x)=v_{\xi}(x)$. The induced momentum and co-momentum maps

$$
\begin{aligned}
\mu^{\prime}: M & \rightarrow\left(\mathrm{g}^{\prime}\right)^{*}=\mathrm{g}^{*} \oplus \mathbf{R} \\
\tilde{\mu}^{\prime}: \mathrm{g}^{\prime}=\mathrm{g}+\mathbf{R} & \rightarrow \mathcal{C}^{\infty}(M)
\end{aligned}
$$

satisfy

$$
v_{h_{(\xi, a)}}=v_{h_{\xi}},
$$

where $h_{(\xi, a)}=\tilde{\mu}^{\prime}(\xi, a)$ and $h_{\xi}=\tilde{\mu}(\xi)$. This implies that $h_{(\xi, a)}-h_{\xi}=$ $\varrho(\xi, a)$, with $\varrho(\xi, a)$ constant. The obstruction to the infinitesimal equivariance of $\tilde{\mu}^{\prime}$ is

$$
\begin{aligned}
\Sigma^{\prime}((\xi, a),(\eta, b)) & =\tilde{\mu}^{\prime}([(\xi, a),(\eta, b)])-\left\{\tilde{\mu}^{\prime}(\xi, a), \tilde{\mu}^{\prime}(\eta, b)\right\}= \\
& =\tilde{\mu}^{\prime}([\xi, \eta], \Sigma(\xi, \eta))-\{\tilde{\mu}(\xi)+\varrho(\xi, a), \tilde{\mu}(\eta)+\varrho(\eta, b)\} \\
& =\tilde{\mu}([\xi, \eta])+\varrho([\xi, \eta], \Sigma(\xi, \eta))-\{\tilde{\mu}(\xi), \tilde{\mu}(\eta)\} \\
& =\Sigma(\xi, \eta)+\varrho([\xi, \eta], \Sigma(\xi, \eta)) .
\end{aligned}
$$

The 2-cocycle $\Sigma^{\prime}((\xi, a),(\eta, b))$ belongs to the trivial class if it is a coboundary, i.e. if $\Sigma^{\prime}((\xi, a),(\eta, b))=-\lambda([(\xi, a),(\eta, b)])=-\lambda([\xi, \eta], \Sigma(\xi, \eta))$. Let's define the 1-cochain $\tau: \mathrm{g}^{\prime} \rightarrow \mathbf{R}$ given by $\tau(\xi, a)=a$. Then $\Sigma(\xi, \eta)=\tau([\xi, \eta], \Sigma(\xi, \eta))$ and thus

$$
\begin{aligned}
\Sigma^{\prime}((\xi, a),(\eta, b)) & =(\tau+\varrho)([\xi, \eta], \Sigma(\xi, \eta)) \\
& =(\tau+\varrho)([(\xi, a),(\eta, b)]) \\
& =-\lambda([(\xi, a),(\eta, b)]),
\end{aligned}
$$

with $\tau+\varrho=-\lambda$. Therefore, the co-comentum map $\tilde{\mu}^{\prime}$ can be adjusted to be infinitesimally equivariant by choosing $\varrho(\xi, a)=-a$. The resulting co-momentum map is then

$$
\tilde{\mu}^{\prime}(\xi, a)=\tilde{\mu}(\xi)-a
$$


We will now consider the action of $\mathrm{g}=\mathbf{R}^{2}=\{(a, b)\}$ on $M=$ $\mathbf{R}^{2}=\{(q, p)\}$ given by $\Phi_{(a, b)}(q, p)=(q+a, p+b)$. The realization of the Lie algebra element $\xi=(a, b)$ is given by the fundamental vector field $v_{\xi}=a \partial_{q}+b \partial_{p}$. This vector field is the Hamiltonian vector field corresponding to the function $h_{\xi}(q, p)=a p-b q$. The co-momentum map is then

$$
\begin{aligned}
\tilde{\mu}: \mathrm{g} & \rightarrow \mathcal{C}^{\infty}(M) \\
(a, b) & \mapsto h_{\xi}(q, p)=a p-b q .
\end{aligned}
$$

This co-momentum map is not infinitesimally equivariant. This means that it is not a homomorphism of Lie algebras. The obstruction $\Sigma$ is given by

$$
\begin{aligned}
\Sigma\left(\left(a_{1}, b_{1}\right),\left(a_{2}, b_{2}\right)\right) & =\tilde{\mu}\left(\left[\left(a_{1}, b_{1}\right),\left(a_{2}, b_{2}\right)\right]\right)-\left\{\tilde{\mu}\left(a_{1}, b_{1}\right), \tilde{\mu}\left(a_{2}, b_{2}\right)\right\} \\
& =b_{1} a_{2}-a_{1} b_{2} .
\end{aligned}
$$

Since $[\mathrm{g}, \mathrm{g}]=0$, the only coboundary is zero. This means that $\Sigma$ belongs to a non-trivial cohomology class. In particular let's consider the Lie algebra elements $\xi_{q}=(1,0)$ and $\xi_{p}=(0,1)$. These elements induce on $M$ the fundamental vector fields $v_{\xi_{q}}=\partial_{q}$ and $v_{\xi_{p}}=\partial_{p}$ respectively. In this case the obstruction is

$$
\begin{aligned}
\Sigma\left(\xi_{q}, \xi_{p}\right) & =\tilde{\mu}\left(\left[\xi_{q}, \xi_{p}\right]\right)-\left\{\tilde{\mu}\left(\xi_{q}\right), \tilde{\mu}\left(\xi_{p}\right)\right\} \\
& =\tilde{\mu}(0)-\{p,-q\} \\
& =-1
\end{aligned}
$$

Since the action of $\mathrm{g}=\mathbf{R}^{2}$ on $M=\mathbf{R}^{2}$ is not infinitesimally equivariant, classical mechanics on $M$ is not a satisfactory realization of the universal model $\mathrm{g}^{*}$ for the $G$-action. It is worth noting that the nonequivariance of $\tilde{\mu}$ is a consequence of the fact that the algebraic structure of $\mathrm{g}$ does not reflect the symplectic intertwining between physical properties. In other words, $\left[\xi_{q}, \xi_{p}\right]=0$ even if $\{q, p\}=1$. The infinitesimally equivariant co-momentum map is obtained through the central extension of $\mathrm{g}=\mathbf{R}^{2}$ defined by $\Sigma$. It is given by

$$
\begin{aligned}
\tilde{\mu}^{\prime}: \mathrm{g}^{\prime}=\mathbf{R}^{2} \oplus \mathbf{R} & \rightarrow \mathcal{C}^{\infty}(M) \\
((a, b), c) & \mapsto h_{(a, b)}-c=a p-b q-c .
\end{aligned}
$$

The central extension $\mathrm{g}^{\prime}=\mathbf{R}^{2} \oplus \mathbf{R}$ is called Heisenberg algebra and the corresponding connected and simply-connected Lie group is the 
Heisenberg group $H$. The underlying manifold is $H=\mathbf{R}^{2} \times S^{1}$ (where $\mathbf{R}^{2}$ is considered an additive Lie group), with multiplication given by the expression

$$
\left(g_{1}, e^{i \theta_{1}}\right) \cdot\left(g_{2}, e^{i \theta_{2}}\right)=\left(g_{1}+g_{2}, e^{i\left[\theta_{1}+\theta_{2}+\frac{1}{2} \Sigma\left(g_{1}, g_{2}\right)\right]}\right),
$$

with identity $(0,1)$ and inverse $\left(g, e^{i \theta}\right)^{-1}=\left(-g, e^{-i \theta}\right)$.

As we have argued above, adopting postulate $\ddagger$ makes it impossible to identify the space of states with the phase space $M$ (since classical states $x \in M$ are overdetermined). We will now provide some heuristic justifications for the election of a new space of states. If certain conditions were satisfied, it would be possible to use the momentum map to give a rigorous definition of physical properties. More precisely, if the moment map were equivariant - and, as we will see in the next section, if the map $\mathcal{C}^{\infty}(M) \rightarrow \mathcal{H}_{M}$ were injective -, the properties $\tilde{\mu}(\xi)=h_{\xi}: M \rightarrow \mathbf{R}$ would specify how different physical states realize differently universal generators $\xi \in \mathrm{g}$. This characterization of physical properties would explain why the correspondence between observables and operators is a fundamental feature of mechanics. The fact that physical properties $h_{\xi}$ induce generators of infinitesimal canonical transformations - through the map $\pi: \mathcal{C}^{\infty}(M) \rightarrow \mathcal{H}_{M}$ - would be a consequence of the fact that $h_{\xi}$ are quantities - defined through the map $\tilde{\mu}: \mathrm{g} \rightarrow \mathcal{C}^{\infty}(M)$ - that specify how the universal generators $\xi \in \mathrm{g}$ are realized by states in $M$. The following diagram encompasses both sides of the relationship between observables and operators:

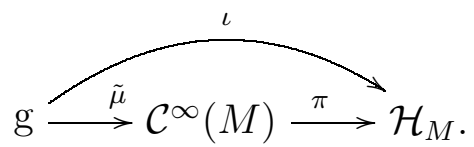

The realization - as Hamiltonian vector fields in $\mathcal{H}_{M}$ - of universal symmetry transformations in $\mathrm{g}$ is factorized through physical properties in $\mathcal{C}^{\infty}(M)$, which can be used for identifying the state. In this way, each physical property defines a realization of a universal infinitesimal canonical transformation that acts on another possible property. The phase space $M$ is then characterized by a set of $2 n$ simplectically intertwined possible properties. In particular, we have considered the transitive action of the translation group $\mathbf{R}^{2}$ on $M=\mathbf{R}^{2}$. The problem we found is that this group does not reflect the symplectic intertwining 
between properties in $M$ (since $\left.\left[\xi_{q}, \xi_{p}\right]=0\right)$. This fact is manifested through the non-equivariance of the corresponding momentum map (i.e. it does not preserve the relevant algebraic structures). In order to guarantee the equivariance of the momentum map, the translation group $\mathbf{R}^{2}$ has to be extended to the Heisenberg group $H=\mathbf{R}^{2} \times S^{1}$. Nevertheless, even if the corresponding Heisenberg algebra reflects the symplectic intertwining between canonically conjugated properties $\left[\left(\xi_{q}, 0\right),\left(\xi_{p}, 0\right)\right]=\left(\left[\xi_{q}, \xi_{p}\right], \Sigma\left(\xi_{q}, \xi_{p}\right)\right)=(0,-1)-$, its action on $M$ is still given by classical operators, i.e. by Hamiltonian vector fields $(\xi, a) \in \mathrm{g}^{\prime}$ acts on $M$ by $\rho(\xi, a)(x)=v_{\xi}(x)-$. As we will see in the next section, the Lie algebra of Hamiltonian vector fields is not isomorphic to the Poisson algebra. This means that it does not reflect the intertwining between coordinates $q$ and momenta $p$. If one wants to define physical properties as quantities that specify how different states realize differently universal operations, this realization must be faithful. In other words, the realized operator algebra has to be isomorphic to the universal operator algebra. This means that non-commuting universal operators should be mapped to non-commuting realized operators. Moreover, it is also necessary to consider the following important factor. Since $(q, p)$ is a complete set of observables - every function that commutes with both $q$ and $p$ is necessarily constant -, the induced (quantum) operators $\hat{v}_{q}$ and $\hat{v}_{p}$ must also be a complete set. This means that any operator commuting with both $\hat{v}_{q}$ and $\hat{v}_{p}$ is necessarily a multiple of the identity. This conditions amounts to demand that the operators $\hat{v}_{q}$ and $\hat{v}_{p}$ act irreducibly on the space of states. ${ }^{12}$ To sum up, the searched space of states has to be endowed with an irreducible action of operators $\hat{v}_{q}$ and $\hat{v}_{p}$, such that their commutator algebra is isomorphic to the Poisson algebra. In the case $M=\mathbf{R}^{2 n}$, the Stone-Von Neumann theorem guarantees all irreducible representations of the Heisenberg algebra to be unitary equivalents to the Schrödinger representation (for a fixed value of Planck's constant). In this representation, operators associated to the observables $q$ and $p$ act on functions in $L^{2}\left(\mathbf{R}^{n}\right)$ by means of the expressions $\hat{v}_{q} \psi(q)=q \psi(q)$ and $\hat{v}_{p} \psi(q)=-i \hbar \frac{\partial \psi(q)}{\partial q}$. It is worth noting that the resulting quantum states satisfy postulate $\ddagger$ by construction. For example, if a quantum state has a well defined

\footnotetext{
${ }^{12}$ Irreducibility means that every closed subspace of the space of states which is invariant under the action of this set is either $\{0\}$ or the whole space of states.
} 
position $-|\psi\rangle=|q\rangle-$, the transformation generated by the operator $\hat{v}_{q}$ (multiplication by $q$ in the coordinate representation) should not be an active transformation between physically distinct states, but rather a symmetry transformation that does not modify the physical state. In fact, the transformed state $e^{i \hat{v}_{q} k}|q\rangle=e^{i q k}|q\rangle$ is gauge equivalent to $|q\rangle$. On the other hand, since the observable $p$ is not an objective property of the state $|q\rangle$, transformations generated by $\hat{v}_{p}$ - i.e. translations in position - should not be considered symmetry transformations, but rather active transformations between physically different states. This is consistent with the fact that $e^{i \hat{v}_{p} k}|q\rangle$ is not gauge equivalent to $|q\rangle$.

\section{From objective Properties to REALIZED SyMmetry TRANSFORMATIONS}

We will now consider the results described in the preceding section from a dual point of view. We have seen that in order to define an equivariant co-momentum map between universal operators and observables it is necessary to enlarge the translation group that acts on $M$ by adding an extra $S^{1}$-degree of freedom (defining in this way the Heisenberg group). Conversely, the prequantization formalism shows that in order to define a faithful correspondence between observables and realized operators it is necessary to enlarge the symplectic manifold $M$ by adding an extra $S^{1}$-degree of freedom ([3], [8], [12], [13], [14]).

A fundamental feature of classical mechanics is that the surjective Lie algebra homomorphism $\mathcal{C}^{\infty}(M) \rightarrow \mathcal{H}_{M}$ - defined by the symplectic structure $\omega$ - between classical observables $f \in \mathcal{C}^{\infty}(M)$ and classical operators $v_{f} \in \mathcal{H}_{M}$ is not injective, being its kernel the set of constant functions $f=k \in \mathbf{R}$. These properties of the map $\mathcal{C}^{\infty}(M) \rightarrow \mathcal{H}_{M}$ can be summed up by saying that the short sequence

$$
0 \longrightarrow \mathbf{R} \stackrel{i}{\longrightarrow} \mathcal{C}^{\infty}(M) \stackrel{\pi}{\longrightarrow} \mathcal{H}_{M} \longrightarrow 0
$$

is exact. In other words, the image of the injection $i$ - the constant functions in $\mathcal{C}^{\infty}(M)$ - is the kernel of the projection $\pi$ (i.e. $v_{f=k}=0$ ). This means that, as Lie algebras, the Poisson algebra of classical observables $\mathcal{C}^{\infty}(M)$ and the Lie algebra $\mathcal{H}_{M}$ of Hamiltonian vector fields are not isomorphic. Consequently, while for exemple the infinitesimal transformation of $q$ generated by $p$ is given by $\mathcal{L}_{i} e_{v_{p}} q=\{q, p\}=1$, the same infinitesimal action applied to $v_{q}$ yields $\mathcal{L} i e_{v_{p}} v_{q}=\left[v_{p}, v_{q}\right]=0$. 
In other words, the classical differential operator $v_{p}$ acts non-trivially on $q$ and trivially on $v_{q}$. This means that classical mechanics fails to establish a faithful correspondence between observables and operators: a transformation of an observable $f$ is not necessarily reflected at the level of the induced operator $v_{f}$. Nevertheless, the definition of a physical property as a quantity that specifies how different states realize differently universal operations is consistent only if a change in the value of the property is faithfully reflected in the corresponding realized operators. It is worth noting that $\left[v_{p}, v_{q}\right]=0$ (while $\{q, p\}=1$ ) is the dual version of the fact - considered in the preceding section that $\left[\xi_{p}, \xi_{q}\right]=0$ (while $\left\{\tilde{\mu}\left(\xi_{p}\right), \tilde{\mu}\left(\xi_{q}\right)\right\}=\{q, p\}=1$ ). In other words, both the universal operator algebra $g$ and the realized operator algebra $\mathcal{H}_{M}$ fail to reflect the symplectic intertwining defined by the Poisson algebra between properties that are canonically conjugated.

The geometric arena of classical mechanics is insufficient for establishing a faithful correspondence between observables and operators. As the prequantization formalism shows, in order to define an operator algebra isomorphic to the Poisson algebra $\mathcal{C}^{\infty}(M)$, it is necessary to extend classical operators $v_{f}$ to quantum operators $\hat{v}_{f}$ by adding additional components to the former. To do so, the symplectic manifold $M$ has to be extended by defining a complex line bundle over it. The quantum operators $\hat{v}_{f}$ must satisfy the quantization conditions proposed by Dirac [4]:

- $\left.Q_{1}\right)$ If $f=k \in \mathbf{R}$, then $\hat{v}_{f=k}=k I$, where $I$ is the identity operator.

- $\left.Q_{2}\right)$ If $\{f, g\}=h$, then $\left[\hat{v}_{f}, \hat{v}_{g}\right]=-i \hbar \hat{v}_{h}$.

- $\left.Q_{3}\right)$ If $\left\{f_{i}\right\}$ is a complete set of classical observables, then the Hilbert space of quantum states has to be irreducible under the action of the set $\left\{\hat{v}_{f_{i}}\right\}$.

The prequantization formalism shows that, in order to define an operator algebra that satisfies $Q_{1}$ and $Q_{2}$, it is necessary to define a complex line bundle $L \rightarrow M$ over the phase space $M$ with a hermitian connection $\nabla$, such that its curvature is given by the symplectic form $\omega$. The additional "internal" dimensions defined by the fibers let us extend the classical operators $v_{f} \in \mathcal{H}_{M}$ by means of vertical components $\zeta_{f}$ tangent to the fibers. By construction, the extended quantum operators $\hat{v}_{f}=v_{f}^{h}+\zeta_{f}$ (where $v_{f}^{h}$ is the horizontal lift of $v_{f}$ 
defined by the connection) satisfy commutation relations isomorphic to a subalgebra of classical observables' Poisson algebra. This guarantees that $\left[\hat{v}_{q}, \hat{v}_{p}\right] \neq 0$, even if $\left[v_{q}, v_{p}\right]=0$. By adding an internal "quantum" dimension to the symplectic manifold $M$, it is possible to define a quantum operator algebra isomorphic to the Poisson algebra. It can then be shown that the differential operators $\hat{v}_{f}$ acts on sections $s: M \rightarrow L$ by means of the expression [3]

$$
\hat{v}_{f} \cdot s=\nabla_{v_{f}} s-f s .
$$

Therefore, it might seem natural to identify the sections $s: M \rightarrow L$ with the new notion of physical states. Nevertheless, since these sections depend on the $2 n$ coordinates of the phase space $M$, they do not correspond to the usual notion of quantum states, which only depends on $n$ coordinates. In fact, if quantum states were defined by these sections, they could be localized in both $q$ and $p$. This means that both $q$ and $p$ could be objective properties of the physical state. However, as explained above, such simultaneous localization would be contradictory with postulate $\ddagger$. The formal counterpart to this conceptual objection is that the quantum operators associated to a complete set of classical observables by the prequantification formalism, is not a complete set (they do not act irreducibly on the space of quantum states [7]). ${ }^{13}$ This problem can be solved by restricting the space of quantum states. To do so, it is necessary to perform a second stage in the quantization process given by the election of a polarization (i.e. a foliation of $M$ by Lagrangian submanifolds [14]). The resulting polarized quantum states only depend on the $n$ coordinates of a Lagrangian submanifold of $M$. This means that they cannot be localized in both $q$ and $p$.

In this way the geometric quantization formalism allow us to reobtain the results described in the preceding section. In order to define physical properties by means of an equivariant realization of universal operations, it is necessary to extend the translation group in $M$ to

\footnotetext{
${ }^{13}$ For example, the prequantization of the cotangent bundle $M=T^{*} \mathbf{R}$ associates to the complete set of canonical variables $q$ and $p$ the quantum operators $\hat{v}_{q}=$ $q+i \hbar \frac{\partial}{\partial p}$ and $\hat{v}_{p}=-i \hbar \frac{\partial}{\partial q}$ respectively. Let's consider the subset $\mathcal{C}^{\infty}(\mathbf{R}) \subset \mathcal{C}^{\infty}(M)$ composed of states of the form $\psi(q)$. The transformed states $\hat{v}_{q} \psi(q)=q \psi(q)$ and $\hat{v}_{p} \psi(q)=-i \hbar \frac{\partial \psi(q)}{\partial q}$ also depend only on $q$. This means that $\mathcal{C}^{\infty}(\mathbf{R})$ is a proper subspace of quantum states invariant under the action of the quantum operators $\hat{v}_{q}$ and $\hat{v}_{p}$.
} 
the Heisenberg group. The Stone-Von Neumann theorem assures that there is essentially a unique irreducible representation of this group, which is given (modulo unitary equivalence) by the Schrödinger representation. On the other hand, in order to define an operator algebra that is isomorphic to the Poisson algebra, it is necessary to extend the symplectic manifold by defining a fiber bundle over it. The corresponding operator algebra acts irreducibly on the space of polarized sections. By choosing a vertical polarization the Schrödinger representation is recovered [14].

\section{Conclusion}

Beyond the empirical observation that quantum mechanics is, at least to our present knowledge, the correct theory of mechanics, it still lacks a satisfactory explanation of the rational necessity of substituting classical mechanics by quantum mechanics. In this paper we proposed that an analysis of the relations between objective properties of physical states and symmetry transformations could shed new light on this problem. The twofold role played by classical observables in mechanics - as functions that can be evaluated on states and as generators of canonical transformations - is considered here a fundamental feature that deserves further attention. Our first observation is that the classical correspondence between these two roles is not satisfactory. This can be seen in two ways. On the one hand, the map $\mathcal{C}^{\infty}(M) \rightarrow \mathcal{H}_{M}$ between classical observables and Hamiltonian vector fields is not injective. On the other hand, the co-momentum map $\tilde{\mu}: \mathrm{g} \rightarrow \mathcal{C}^{\infty}(M)$ is not equivariant (where $\mathrm{g}$ is the Lie algebra of the translation group in $M$ ). This means that the Lie algebras $\mathcal{H}_{M}$ and $g$ do not reflect the symplectic intertwining between properties that are canonically conjugated. In fact, quantum mechanics can be understood as the theory of mechanics that bypasses these flaws. Dirac's quantization conditions can be considered a formalization of this prescription. The geometric quantization formalism shows that in order to satisfy Dirac's conditions (at least for a certain subalgebra of observables), classical operators (Hamiltonian vector fields) have to be extended by adding new "vertical" components. In order to define these new components it is necessary to extend the phase space $M$ by defining a complex line bundle over it (endowed with a hermitian connection of curvature defined by the symplectic form). Quantum states are then given by polarized 
sections of the line bundle. From the dual point of view, in order to define an equivariant momentum map, the translation group in $M$ has to be extended to the Heisenberg group. For $M=\mathbf{R}^{2 n}$, the StoneVon Neumann theorem fixes (modulo unitary equivalence) its unique irreducible representation.

These formal results show that, to a certain extent, quantum mechanics can be recovered by forcing a faithful - or equivariant - correspondence between observables and operators. Hence, in order to understand the necessity of quantum mechanics, it is necessary to better establish the rational link between the two roles played by observables. As a means of establishing this rational link, we proposed postulate ‡. According to this postulate, the infinitesimal canonical transformations induced by the objective properties of a physical state should not be considered active transformations between different physical states - as in classical mechanics -, but as symmetry transformations that do not affect its objective properties, i.e. that do not modify the physical state. This implies that states connected by these transformations have to be considered gauge equivalent. In other words, the objective properties that allow us to identify the state define also the symmetry transformations that gauge its non-objective properties. Moreover, the momentum map formalism permits a better understanding of the relationships between observables and operators. According to this formalism, the objective properties of a physical state characterize the way the state realizes equivariantly certain universal transformations. Such considerations give a conceptual scope to the correspondence between observables and operators. Nevertheless, we showed that classical mechanics cannot be consistently interpreted by means of postulate $\ddagger$. In fact, a determination of classical states by both $q$ and $p$ is inconsistent with the interpretation of the induced infinitesimal canonical transformations as effective symmetry transformations. According to the proposed postulate, since each objective property characterizes the realization of a symmetry transformation that gauges the canonically conjugated property, not all properties can be consistently attributed to a particular physical state. This implies that classical states are overdetermined, that is to say that they are defined by means of too many canonical variables. On the contrary, the quantum uncertainty principle can be considered a faithful realization of postulate $\ddagger$. The momentum of a state with a well defined position $q$ is completely gauged 
out by means of the symmetry transformation induced by the observable $q$ (which is an objective property of the state). Therefore, we can conclude that it is not the case that the quantum notion of physical objectivity is underdetermined - when compared to a hypothetical classical completeness - but that the classical notion is overdetermined. It is a remarkable fact that non-commutativity of quantum operators is the condition of possibility for a well-defined notion of physical objectivity. Quantum non-commutativity guarantees that the symplectic intertwining between properties that are canonically conjugated is faithfully reflected at the level of operators. Therefore, physical properties of states can be consistently defined as the quantities that specify faithfully how different states realize different universal operations.

\section{Acknowledgements}

This work was partially supported by the grants PICT 04-17687 (ANPCyT), PIP No 1478/01 (CONICET), UBACyT No X204 and the financial support of Fondation Maison des Sciences de l'Homme (Paris).

\section{REFERENCES}

[1] Abraham, R., Marsden, J.E.: Foundations of Mechanics, second ed., AddisonWesley Publishing Company (1978)

[2] Arnold, V.I.: Mathematical Methods of Classical Mechanics, Springer, New York (1989)

[3] Brylinski, J.-L.: Loop Spaces, Characteristic Classes, and Geometric Quantization, Progr. Math. 107, Birkhäuser Boston Inc., Boston MA (1993)

[4] Dirac, P.A.M.: The Principles of Quantum Mechanics, revised fourth ed., Oxford Univ. Press, Oxford (1967)

[5] Goldstein, H.: Classical Mechanics, Addison-Wesley Publishing Company (1981)

[6] Isham, C.J.: Topological and Global Aspects of Quantum Theory. In: DeWitt, B.S., Stora, R. (eds.) Relativity, Groups and Topology II, Les Houches, Session XL, Elsevier Science Publishers B.V. (1984)

[7] Kirillov, A.A.: Lectures on the Orbit Method, Graduate Studies in Mathematics, Vol. 64, AMS (2004)

[8] Kostant, B.: Quantization and Unitary representations, Lecture Notes in Mathematics, Vol. 170, Springer-Verlag, Berlin-Heidelberg-New York (1970)

[9] Libermann, P., Marle, C.-M.: Symplectic Geometry and Analytical Mechanics, D. Reidel Publishing Company, Holland (1987) 
[10] Marsden, J.E., Ratiu, T.S.: Introduction to Mechanics and Symmetry, second ed., Springer-Verlag, New York (1999)

[11] Ortega, J.-P., Ratiu, T.S.: Momentum maps and Hamiltonian Reduction, Birkhäuser Boston (2004)

[12] Puta, M.: Hamiltonian Mechanical Systems and Geometric Quantization, Kluwer Academic Publishers (1993)

[13] Souriau, J.-M.: Structure of Dynamical Systems. A Symplectic View of Physics, Birkhäuser Boston (1997)

[14] Woodhouse, N.: Geometric Quantization, second ed., Oxford University Press, Oxford (1992) 